\title{
El fútbol profesional y su responsabilidad social corporativa en Cataluña (2006-2010) Mucho más que Unicef
}

\author{
Xavier Ginesta (Universitat de Vic) \\ Enric Ordeix (Universitat Ramon Llull)
}

Recibido: 3/9/2011

Aprobado: 30/10/2011

\begin{abstract}
REsumen: Las fundaciones son una forma de potenciar la responsabilidad social corporativa (RSC) de las organizaciones. En Cataluña, el FC Barcelona, el RCD Espanyol o la Federación Catalana de Fútbol (FCF) crearon sus fundaciones para expresar su filosofía y transmitir sus valores en la sociedad. Las fundaciones que hemos estudiado son referencia en lo deportivo y empresarial en Cataluña, sobre todo gracias a los proyectos articulados entre los años 2006 y 2010, algunos de ellos con la intención de internacionalizar las marcas, los clubes y la ciudad.
\end{abstract}

Palabras clave: Fundaciones / fútbol catalán / responsabilidad social corporativa / organizaciones deportivas / relaciones públicas

Professional soccer and its corporate social responsibility in Catalonia. Much more than Unicef

Summary: Foundations are important tolls to promote corporate social responsibility (CSR) for organizations. In Catalonia, FC Barcelona, RCD Espanyol or the Catalan Soccer Federation (FCF) devised their foundations to express their philosophy and transmit their values to society. The foundations we study are benchmarks in the sporting and economic fields in Catalonia, mainly because of the projects they developed between 2006-2010, some of them with the objective of the internationalization of sports brands, clubs and cities.

Key words: Foundations / Catalan football / corporate social responsibility / sport organizations / public relations 


\section{Introducción}

$\mathrm{E}$ n una sociedad globalizada, la comunicación trasciende el mero proceso de la actividad comunicativa. La esencia en que se sustentan las relaciones públicas yace en la identidad y la actitud de las organizaciones. No se puede comprender un proceso comunicativo sin unos valores corporativos sólidos que componen una cultura organizativa en simbiosis con el entorno. Las organizaciones deportivas no están al margen de esta tendencia, sino que son ahora mismo uno de los sectores pioneros en el uso de la responsabilidad social corporativa (RSC) como forma de expresar esta actitud organizativa permeable con lo social.

Hay un debate abierto referente a la creación de actitudes, pues la comunicación debe obedecer a una actitud previa o, por lo contrario, contribuye a la creación de esta actitud. Los autores de este artículo partimos del punto de vista de que sin actitud no hay comunicación y que las relaciones públicas son el sumatorio de ambas, siendo así en relaciones públicas parte implícita de la filosofía de management de las organizaciones.

Las organizaciones deportivas han entendido este principio, pues han buscado, a través de la creación de fundaciones, elementos de interés general que confluyen con sus intereses como organización para así expandir su identidad de marca, bien sea a nivel internacional, como el FC Barcelona, o bien sea para la fidelización de los públicos más locales, como es el caso del RCD Espanyol.

Si bien estamos acostumbrados a percibir que la construcción de la marca se deriva de lo corporativo hacia lo comercial, este principio sucede esencialmente en las empresas con productos de gran consumo. Por lo contrario, las empresas $u$ organizaciones de servicios construyen la marca a partir de valores que la identifican con su entorno de influencia $y$, a partir de allí, determinan su valor añadido en el mercado. Este es el caso de las organizaciones deportivas, dentro del sector de servicios de ocio.

Este artículo, centrado en el análisis de la estructura de comunicación de las principales fundaciones del fútbol profesional catalán hasta el $2010^{1}$ (FC Barcelona, RCD Espanyol y Federació Catalana de Futbol), pretende establecer los indicadores de análisis que determinen la excelencia en la gestión de sus relaciones públicas en el ámbito social.

1 Los proyectos de las fundaciones analizados son los que están comprendidos en el período 2006-2010, último mandato de Joan Laporta al frente del FC Barcelona, de Daniel Sánchez Llibre al frente del RCD Espanyol (2006-2011), y años que abarca la presidencia de Jordi Roche en la FCF. 


\section{Metodología y antecedentes}

La metodología principal de esta investigación ha sido la cualitativa, mediante la entrevista en profundidad con los responsables de comunicación de las tres fundaciones que forman parte de esta muestra tipológica (Marc Parramon, de la Fundació FC Barcelona; Jordi Munté, de la Fundació Privada RCD Espanyol, y Bernat Bufaluy, de la Fundació Privada Catalana de Futbol), estableciendo un cuestionario dirigido por los investigadores que permite cruzar los resultados de las tres entrevistas. Los indicadores de referencia que se tuvieron en cuenta en las preguntas de las entrevistas fueron: estructura (organigrama, vinculación de la fundación con el resto del club); líneas de trabajo y proyectos de las fundaciones; medios propios y técnicas de relaciones públicas; y sinergias sociales. Los datos que se obtuvieron se triangularon con los obtenidos del análisis de sus páginas web $\mathrm{y}$ los servicios de valor añadido que estas ofrecen, y se confrontaron los resultados con las informaciones aparecidas en los periódicos, entendiendo estas informaciones como un elemento de visualización de los proyectos sociales de estas organizaciones.

No obstante, estos resultados no tendrían sentido sin un contraste con las teorías más relevantes del sector de las relaciones públicas y la responsabilidad social corporativa, así como los artículos y estudios académicos que mejor determinan las variables y prin- cipios de la excelencia en la gestión de las relaciones corporativas y en la gestión de los procesos comunicativos que se derivan de ellas, como los de Botan y Hazleton (1989), Grunig (1992), Spicer (1997) y Perdiguero (2003). Sin embargo, teniendo en cuenta que el artículo confluye con aspectos significativos de la estructura y el comportamiento organizacional, así como con los elementos básicos de la gestión del cambio y la negociación, entendemos que sería conveniente tener en cuenta las aportaciones de otros autores como Mintzberg (1995), D’Aprix (1996) o Drucker (2006).

\section{Marco teórico. La gestión de los valores de la organización en la era digital}

La finalidad de las relaciones públicas es conseguir una complicidad significativa con los públicos de las organizaciones, para así tener la máxima adaptabilidad a los intereses de la comunidad y evitar disfunciones en su estrategia de gestión de la confianza. Así pues, la legitimidad de las organizaciones yace en su mayor medida en la capacidad de articular las competencias profesionales referentes a a las habilidades comunicativas, al conocimiento del entorno y a la determinación de las actitudes organizativas. Estos conceptos fueron citados en las conclusiones del Congreso Internacional de Investigadores de Relaciones Públicas, que tuvo lugar 
en la Facultad de Comunicación Blanquerna (Universitat Ramon Llull), en abril del 2009.

Es de comprensión general que las organizaciones, en especial las que tienen un peso social significativo, como las fundaciones deportivas, intenten buscar un mínimo común denominador en lo que a actitudes y valores sociales se refiere, para aumentar la transversalidad de la marca. De esta forma, conceptos como "infancia", "solidaridad", "educación" o "cooperación", los cuatro figurantes entre los principios de las fundaciones analizadas en la muestra tipológica, contribuyen a aumentar la legitimidad de los clubes de donde dependen estas fundaciones por parte de sus públicos. El papel de las relaciones públicas en este proceso es demostrar la capacidad que tiene la entidad de corresponder a estos principios. $\mathrm{Si}$, de un lado, la publicidad y el márketing prometen y la organización se compromete, las relaciones públicas deben preciarse como vehiculadoras y justificadoras de esta promesa y, además, deben desarrollar acciones que les permiten estar presentes en los medios en el debate público como líderes de opinión de estos conceptos.

Para comprender este fenómeno hay que explicar tres cambios significativos en la gestión de los valores de las organizaciones en la nueva era digital: cambios organizativos, cambios comunicativos y cambios actitudinales. En cuanto a los cambios or- ganizativos, ha habido un cambio de paradigma en la tipología de sistemas organizativos fruto de la existencia de los nuevos sistemas de participación. Los sistemas organizativos abiertos distribuyen funciones, responsabilidades y poder entre los miembros de una organización de forma holística, adaptable al entorno y con un claro enfoque a la misión y visión de la entidad (Mintzberg 1995).

En lo referido a los cambios comunicativos, las tecnologías de la información y la comunicación (TIC) (televisión digital, internet y telefonía móvil), han provocado un cambio fundamental en los procesos comunicativos, especialmente en los campos estratégicos, como los recursos humanos, las relaciones públicas y el márketing. Las estructuras comunicativas horizontales, que combinan flujos horizontales $y$ verticales, formales e informales entre los miembros de la organización, obligan a trabajar la viralidad de los mensajes con mucha más precisión y teniendo en cuenta los efectos, positivos o negativos, que las acciones de los públicos puedan tener en la organización, tal y como demuestran los estudios de Freeman (1984), Freeman y McVea (2001) o Inkpen (2001). En el mundo del deporte, hay numerosa bibliografía que hace referencia en cómo el nuevo entorno digital de comunicación ha cambiado los procesos de comunicación de las organizaciones deportivas; por ejemplo, los trabajos de Moragas (2003), Rowe (2003), Helland (2007), Olabe (2008), Lefever, Lievens 
y Valcke (2008) y, finalmente, Ginesta (2009).

Por último, los cambios mencionados previamente actúan a expensas de los substanciosos cambios actitudinales que se están generando en las instituciones y las empresas contemporáneas. Los nuevos sistemas organizativos y los nuevos sistemas comunicativos generan nuevas actitudes de marca y nuevas concepciones en la gestión de la cultura organizativa, y viceversa. La actitud empresarial es una actitud ampliamente más ética (honestidad, transparencia, humanidad, compromiso) que anteriormente. Es decir, ser ético acaba siendo rentable a medio y a largo plazo, pues existe una mayor fidelidad de sus públicos y una mejor construcción de los principios que definen la marca. Los objetivos individuales se alinean con mayor prontitud a los objetivos colectivos y, por consiguiente, los objetivos organizativos de la mayoría de las organizaciones deben alinearse con los objetivos sociales de referencia, tal y como hacen la mayoría de fundaciones deportivas. Como afirma Drucker (1980), "el director de una institución tiene que pensar en las políticas que coinciden con el interés general y provocan cohesión social".

Por lo tanto, uno de los principios básicos de la gestión excelente de las relaciones públicas es equilibrar el interés privado de la organización con el interés de sus públicos y el de la socie-

Figura 1

Cambios en la gestión de los valores de la organización en la era digital

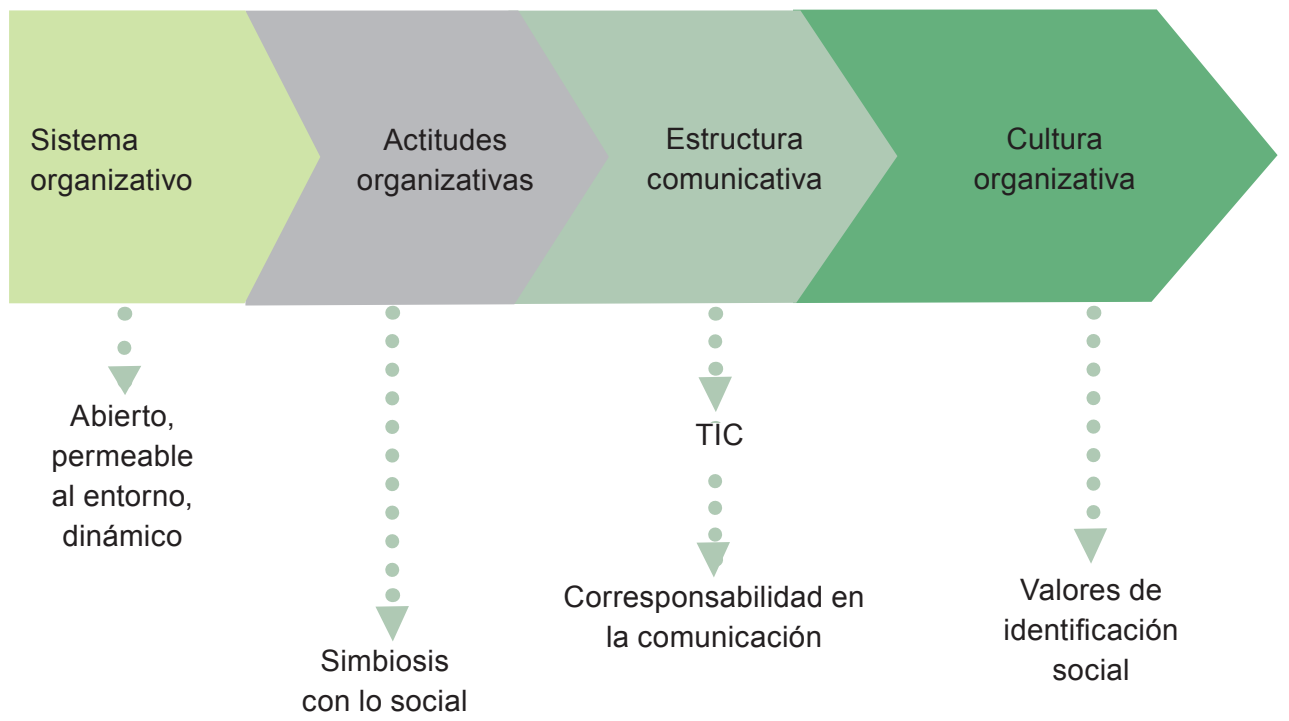


dad. Las relaciones públicas excelentes tienen que planificar estratégicamente y establecer programas de comunicación simétrica, premisa básica para ser socialmente responsables (Grunig 1992: 241).

Las organizaciones modernas están experimentando cambios sustanciales en lo ampliamente conocido como "lo corporativo", entendido como su propia definición como entidad: la misión, la visión, los valores. Tal fenómeno está provocando también un replanteamiento en los roles de gestión de las relaciones públicas y en los objetivos comunicativos concebidos como estratégicos. La cultura de lo inmediato ha trascendido hacia una fidelización del entorno, cosa que ha provocado una mayor sensibilidad hacia la gestión del cambio, tal y como indican Kotter (1998) y Moss (2002). Las relaciones públicas modernas, por lo tanto, tienden a prever y mediar conflictos provocados por dichos cambios. A su vez, buscan el acercamiento con los líderes de opinión y se erigen como tales en su sector y en aquellos conceptos que definen su cultura organizativa, especialmente en los valores que construyen su marca, sea de interés general o no (Ordeix y Duarte 2009). Los clubes se sirven de este acercamiento para marcar más fácilmente la agenda mediática y, por consiguiente, la agenda pública. Por ejemplo, las acciones derivadas del acuerdo entre el FC Barcelona y Unicef (2006) han posicionado al club catalán como un líder en lo que se refiere a los programas de ayuda a la infancia en el Tercer Mundo (Fundació FC Barcelona 2009).

Supervisar y guiar las políticas estratégicas de comunicación para que sean coherentes con las expectativas generadas es otro de los roles esenciales que provienen de los cambios en la gestión de las relaciones públicas. Por lo tanto, conviene que el profesional investigue sobre las tendencias y oportunidades que conllevan los compromisos de la organización así como los riesgos que pueden derivarse de expectativas falsas que no se puedan satisfacer. Por consiguiente, el responsable de relaciones públicas se erige como constructor y velador de la cultura organizativa así como de la gestión comunicativa institucional que haga referencia a los valores de marca. Es generador de opinión y de conciencia de pertenencia en el proyecto organizativo, ya sea público interno como externo. Podríamos dividir, entonces, la gestión de las relaciones públicas en cuatro áreas básicas:

- Área corporativa.- Incluye la gestión de la identidad de la organización a partir de su filosofía organizativa; describe y explica sus políticas de gestión, sus valores organizativos y la actuación de sus órganos de representación.

- Área social.- Incluye las actividades de relaciones públicas sustentadas en principios de interés general, basado en la gestión de temas medioambientales, culturales y de equilibrio social. 
- Área comercial.- Incluye aquellas actividades asociadas de relaciones públicas vinculadas al márketing: presentaciones de producto, acciones de publicity, comunicación viral e interactiva a través de la red, redes de fidelización con los socios y aficionados.

- Área periodística.- Incluye aquellas actividades de proyección social, a través de los medios de comunicación de masas, ya sean acciones asociadas a productos o a temas corporativos.

El cuadro 1 sintetiza las áreas temáticas básicas de gestión de las relaciones públicas que coinciden entre ellas y que en muchas ocasiones comparten técnicas o procedimientos similares. En el caso del ámbito corporativo, la representatividad institucional pue- de trascender a lo social y en el caso comercial, las de acciones de publicity son gestionadas, a menudo, entre las áreas comerciales y de comunicación de las organizaciones, tal y como Ginesta (2009) apuntó en los resultados de su tesis doctoral sobre gestión de la comunicación en el fútbol español.

En conclusión, la gestión de las relaciones públicas son de vital importancia para la gestión de la personalidad corporativa: pone en contacto a las organizaciones con los líderes de opinión reconocidos en ámbitos de interés compartidos; buscan la legitimidad de las organizaciones para aumentar la representatividad de valores o conceptos y, finalmente, inciden en la dimensión social de la entidad gracias a la convicción e implicación de personas vinculadas. Por consiguiente, vamos a analizar los datos del trabajo

Cuadro 1

Esferas de gestión de las relaciones públicas

\begin{tabular}{|c|c|}
\hline Área comercial & Área corporativa \\
\hline $\begin{array}{l}\text { Comunicación de márketing } \\
\text { Relaciones públicas de producto } \\
\text { Eventos con celebridades } \\
\text { Red de fidelización con socios y aficionados } \\
\text { Acciones de publicity }\end{array}$ & $\begin{array}{l}\text { Políticas corporativas } \\
\text { Valores de marca } \\
\text { Directrices de comunicación } \\
\text { Relaciones institucionales }\end{array}$ \\
\hline Área periodística & Área social \\
\hline $\begin{array}{l}\text { Acciones de publicity } \\
\text { Gabinetes de prensa } \\
\text { Gestión de medios propios } \\
\text { Creación de contenidos }\end{array}$ & $\begin{array}{l}\text { Mecenazgo y patrocinio social y cultural } \\
\text { Responsabilidad social corporativa } \\
\text { Relaciones con la comunidad } \\
\text { Márketing social }\end{array}$ \\
\hline
\end{tabular}


de campo (2006-2010) a partir del modelo teórico planteado en el cuadro 1 . En ello, incidiremos en el tipo de mensajes, el tipo de técnicas y el tipo de públicos que conforman la estrategia comunicativa y de relaciones públicas en las tres fundaciones deportivas que forman parte de la muestra.

\section{Presentación de los casos: Fundaciones y fútbol en Cataluña}

Las fundaciones deportivas españolas tuvieron un espectacular aumento durante la década de 1995-2004 -sobre todo a partir del año 2002, con la nueva Ley 50/2002, de Fundaciones-. Fueron 159 las fundaciones que se crearon, contrastando con las 19 fundaciones creadas en la década anterior (Arévalo 2006). Precisamente, el FC Barcelona creó su fundación en 1994, a través de la Asociación pro Fundación Club Barcelona, y el Real Club Deportivo Espanyol lo hizo en 1997, mediante un colectivo de personas (Arévalo 2006), mientras que la Federació Catalana de Futbol lo hizo en el 2006, en concordancia con la nueva junta directiva de Jordi Roche. Esta dejó de tener actividad en el 2009, coincidiendo con la nueva directiva de Jordi Casals y la reestructuración del ente federativo. ${ }^{2}$ Por este motivo, mientras los datos de las fundaciones de los dos clubes tenían vigencia en el 2009, los de la
Fundació Privada Catalana de Futbol fueron recopilados del trabajo de campo (2006-2008) de la tesis doctoral de Xavier Ginesta (2009).

\section{Estructura comunicativa y proyectos de las fundaciones}

Aunque algunos estudios (Arévalo 2006) apuntan que no hubo una relación directa en el momento de su creación entre el FC Barcelona y su fundación y el RCD Espanyol con la suya, el organigrama de estas fundaciones no deja lugar a dudas sobre la vinculación entre las estructuras del club y las de la fundación. Entre otras cosas, y atendiendo al objeto de estudio de este artículo, tanto los dos clubes como la Federación han hecho que los departamentos de Comunicación y Relaciones Públicas sean los mismos para ambas organizaciones. En los tres casos, la actividad de relaciones públicas se limita a tener un carácter meramente técnico y asociado al protocolo de organización de actos. La vertiente estratégica de esta profesión está incluida, en los tres casos, en las funciones del Departamento de Comunicación, que en la gestión de contenidos y en la proyección pública del mensaje asumen un rol jerárquicamente superior.

En el caso del Espanyol, aun es más significativa la vinculación entre club y fundación, si se considera que no

2 Información obtenida en la entrevista personal con el jefe de prensa de la Federació Catalana de Futbol, Bernat Bafaluy, realizada el 22 de octubre del 2009. 
solo se cruzan sinergias en lo que se refiere a la estructura de comunicación, sino también en lo relativo a la parcela deportiva, teniendo en cuenta que la fundación del club blanco-y-azul tiene en la gestión del programa educativo del fútbol base, una de las principales razones de ser. ${ }^{3}$ Para resumir las actividades de las fundaciones que forman parte de la muestra, se presenta a continuación un cuadro que sintetiza los diversos proyectos que estas llevan

\section{Cuadro 2}

Clasificación de los proyectos sociales de las fundaciones deportivas catalanas

\begin{tabular}{|c|c|c|c|c|}
\hline & Formación & Civismo & Cooperación & Cultura \\
\hline $\begin{array}{l}\text { Fundació FC } \\
\text { Barcelona }^{1}\end{array}$ & $\begin{array}{l}\text { - XICS (Red } \\
\text { Internacional } \\
\text { de Centros } \\
\text { Solidarios) } \\
\text { - JES (Jornadas } \\
\text { de Deporte } \\
\text { Solidario) }\end{array}$ & $\begin{array}{l}\text { - Recurso for- } \\
\text { mativo on line } \\
\text { "Juégala" } \\
\text { - Programa "Fem } \\
\text { equip, fem ciutat" } \\
\text { - Torneo para in- } \\
\text { migrantes "Tot } \\
\text { colors" }\end{array}$ & $\begin{array}{l}\text { - XICS (Red } \\
\text { Internacional } \\
\text { de Centros } \\
\text { Solidarios) } \\
\text { - Programas de } \\
\text { colaboración con } \\
\text { las Naciones } \\
\text { Unidas (Unesco, } \\
\text { Unicef y Acnur) }\end{array}$ & $\begin{array}{l}\text { Premios } \\
\text { Vázquez } \\
\text { Montalbán } \\
\text { - Programa } \\
\text { de fomento } \\
\text { de la cultura } \\
\text { "Lletres al } \\
\text { camp" }\end{array}$ \\
\hline $\begin{array}{l}\text { Fundació } \\
\text { Privada RCD } \\
\text { Espanyol }^{2}\end{array}$ & $\begin{array}{l}\text { - XEF (Red de } \\
\text { Escuelas de } \\
\text { Fútbol) } \\
\text { - Forum Fundació } \\
\text { - Convenio con } \\
\text { universidades } \\
\text { (CEU Abad Oliva) } \\
\text { - Campus } \\
\text { Espanyol } \\
\text { English\&Football }\end{array}$ & $\begin{array}{l}\text { - Circulo de } \\
\text { Amigos del } \\
\text { Espanyol }\end{array}$ & & $\begin{array}{l}\text { - Edición de li- } \\
\text { bros y vídeos }\end{array}$ \\
\hline $\begin{array}{l}\text { Fundació } \\
\text { Privada } \\
\text { Catalana de } \\
\text { Futbol }^{3}\end{array}$ & & & $\begin{array}{l}\text { Apadrinamiento } \\
\text { de jugadores }\end{array}$ & \\
\hline
\end{tabular}

1 FC Barcelona. Fundació. <http://www.fcbarcelona.cat/web/Fundacio/catala/>. [Consulta: 27 de diciem bre del 2009].

2 RCD Espanyol Fundació [en línea]. http://www.rcdespanyol.cat/. [Consulta: 27 de diciembre del 2009].

3 Información obtenida en la entrevista personal con el jefe de Prensa de la Federació Catalana de Futbol, Bernat Bafaluy, realizada el 22 de octubre del 2009.

3 Información obtenida en la entrevista personal con el secretario de la Fundació Privada RCD Espanyol, Jordi Munté, realizada el 5 de noviembre del 2009. 
a cabo, siendo estos proyectos elementos generadores del mensaje que dan valor a la marca deportiva del club.

A partir de este cuadro podemos considerar que el principal punto de encuentro entre las actividades de las fundaciones está en el ámbito de la formación, asociada al desarrollo de los valores cívicos. No obstante, desde el 2006 y el nuevo impulso de la Fundació FC Barcelona, los acuerdos de cooperación internacional con las agencias de las Naciones Unidas han centrado la actividad de esta fundación. Es destacable ver cómo la actividad cultural queda en un plano residual, por lo que el elemento básico de excelencia en la gestión de las relaciones públicas acuñado por Grunig (1992) de sinergia con el entorno se limita a la promoción de valores más universales, como los relativos a la solidaridad y el civismo, en lugar de promocionar valores de tipo más local, como podrían ser los culturales.

\section{Las fundaciones como actores comunicativos: Los medios propios}

La proyección social de las iniciativas que se plasman en el cuadro 2 no sería posible sin que estas fundaciones recibieran el apoyo de los departamentos de comunicación y relaciones públicas de los clubes y la Federación. En este sentido, hay que tener en cuenta los cambios sustanciales marcados en la figura 1 de este artículo, donde se refleja la importancia de las nue- vas tecnologías en las estructuras de comunicación de las organizaciones. Actualmente, la aplicación de las TIC en la gestión de la comunicación ha permitido a las organizaciones crear un área de contenidos propia (a las tradicionales publicaciones corporativas, se le ha unido la web o la televisión digital) suficientemente importante para no depender exclusivamente de la opinión emitida por los medios de comunicación sociales y tener un mayor control de los contenidos publicados.

En el cuadro 3 se detallan los medios de comunicación propios que utilizan las fundaciones analizadas para transmitir sus contenidos; se recuerda que estos medios son los mismos que utiliza la comunicación oficial del club o de la federación. Por este motivo, los profesionales encargados de gestionar la comunicación de estas fundaciones están integrados en el mismo departamento de comunicación del FC Barcelona (38 personas), RCD Espanyol (5 personas) y Federació Catalana de Futbol (4 personas) (Ginesta 2009).

Desde un punto de vista de bidireccionalidad y representatividad pública, como primer paso para desarrollar un análisis de sinergias sociales de las fundaciones, podemos constatar que la Fundació FC Barcelona ha construido un espacio de influencia superior $y$ de receptividad del entorno mayor respecto a las otras dos fundaciones analizadas. Sirva de ejemplo el caso de Barça TV, que como factoría de contenidos para otras televisiones durante 


\section{Cuadro 3}

Medios propios de las fundaciones (2006-2010)

\begin{tabular}{|c|c|c|}
\hline $\begin{array}{l}\text { Fundació FC Barcelona/ } \\
\text { FC Barcelona }\end{array}$ & $\begin{array}{l}\text { Fundació privada RCD } \\
\text { Espanyol/RCD Espanyol }\end{array}$ & $\begin{array}{l}\text { Fundació privada } \\
\text { Catalana de Futbol/FCF }\end{array}$ \\
\hline $\begin{array}{l}\text { Web (Barça TV Online, } \\
\text { Radio B@rça) } \\
\text { Canal YouTube } \\
\text { Boletín electrónico } \\
\text { Barça TV } \\
\text { Servicios a móviles } \\
\text { Revista Barça } \\
\text { Barça Júnior } \\
\text { Barça Camp Nou } \\
\text { FCBusiness }\end{array}$ & $\begin{array}{l}\text { Web (Espanyol TV) } \\
\text { Boletín electrónico } \\
\text { Servicios a móviles } \\
\text { Revista RCDE } \\
\text { Pericos Matx } \\
\text { Infoacció }\end{array}$ & $\begin{array}{l}\text { Web } \\
\text { Revista Temps Afegit } \\
\text { Revista Futbol Fundació }\end{array}$ \\
\hline
\end{tabular}

el período analizado, enviaba semanalmente seis horas de contenidos del FC Barcelona a 144 clientes a nivel internacional, cubriendo casi todas las áreas del planeta (Ginesta 2009).

Otro concepto destacable del cuadro 3, y relacionado con la idea de la proyección internacional de los mensajes, es el uso intachable que estas fundaciones hacen de los servicios TIC. Su importancia radica en dos aspectos: la internacionalización de la organización y la eficiencia en la gestión del cambio.

En primer lugar, estas fundaciones tienen en las páginas web y sus servicios derivados (informativos, sociales, formativos y de relaciones públicas) la herramienta más eficiente para su proyección global y, a la vez, local. De todos modos, solo la Fundació Privada Catalana de Fútbol, mientras estuvo activa (2006-2009), tenía una página web para ella, al margen de la web oficial de la Federación: <www.fundaciofcf.cat $>$. Las otras dos fundaciones vinculadas a clubes comparten un espacio dentro de la web oficial del FC Barcelona (<www.fcbarcelona.cat $>$ ) y del RCD Espanyol (<www.rcdespanyol. cat>), respectivamente. Las dos secciones de las web de los clubes destinadas a la fundación son principalmente informativas, con espacios destinados a explicar los diversos proyectos presentados en el cuadro 2 e ilustrados, en algunos casos, con imágenes estáticas o vídeos de producción propia. Del análisis del cuadro 3 se deriva que la integración multimedia de las web de FC Barcelona y Espanyol es un hecho, sobre todo teniendo en cuenta que los dos clubes han desarrollado su propia área de contenidos audiovisuales, que tiene su proyección a la red a través de los servicios de vídeo bajo demanda Barça TV Online y Espanyol TV. En 
cuanto a los usuarios, destaca el dominio absoluto del FC Barcelona, con 26,3 millones de páginas vistas al mes, contra los 4 millones de páginas vistas al mes del RCD Espanyol (Ginesta 2009).

En segundo lugar, ya en el concepto de excelencia en la gestión de las relaciones públicas, se establece la necesidad de corresponder a la demanda de los públicos de forma simétrica. El uso de las TIC permite una mayor gestión de las demandas comunicativas derivadas de la gestión del cambio organizativo y de los principios derivados de la adaptación a los fundamentos de la gestión del conocimiento.

Para terminar con la exposición del cuadro 3, cabe una anotación sobre la importancia de las revistas oficiales para la transmisión de valores asociados a los proyectos de las fundaciones. En este tipo de publicaciones, las fundaciones analizadas han encontrado un medio para publicar aquellas informaciones, usando el género de reportaje, que "generalmente no tienen interés para la prensa diaria", 4 ya que se alejan del conflicto tradicional intrínseco del mundo del fútbol y apuestan por contenidos más sociales. Estos son los reportajes que Bastenier (2001) concebirá como "reportajes de color, al servicio del ocio del lector". La FCF desarrolló su propia publicación para la fundación, Fútbol Fundació (3.000 ejemplares de tirada, bianual), como publicación para promocionar los proyectos de la fundación, sobre todo el de apadrinamiento de jugadores entre el tejido industrial catalán. De todos modos, esta publicación solo tuvo dos números. El FC Barcelona apostó por el formato monográfico de su revista oficial Barça (133.000 ejemplares de tirada, bimestral) para difundir algunos de los proyectos más importantes de la fundación, como el proyecto Més, que contó con la colaboración de la Acnur y Nike, para "fomentar la educación a través del deporte entre jóvenes vulnerables y refugiados de todo el mundo". Finalmente, el Espanyol también dedica una página de su publicación oficial RCDE (25.000 ejemplares de tirada, cuatrimestral) a los proyectos de la Fundació Privada Catalana de Futbol.

\section{Fundaciones y celebridades: Una relación de simbiosis}

Las fundaciones vinculadas a los clubes de fútbol tienen un activo especialmente importante, que contribuye a la proyección pública de sus proyec-

4 Información obtenida en la entrevista personal con el responsable de Comunicación de la Fundació FC Barcelona, Marc Parramon, realizada el 26 de octubre del 2009.

5 FC Barcelona. "Més, un nuevo reto solidario". <http://www.fcbarcelona.cat/web/castellano/ noticies/club/temporada08-09/12/n081204106395.html>. [Consulta: 27 de diciembre del 2009]. 
tos y valores fundacionales: los futbolistas. La proyección pública que estos deportistas han conseguido a nivel mundial, siendo considerados marcas a título individual, tal como han estudiado Parry (2003), Lessinger (2008) y Ginesta (2009), hace que las fundaciones del FC Barcelona y el RCD Espanyol puedan utilizar su magnetismo como herramienta para conseguir sus fines, más allá de las tradicionales visitas de deportistas a los hospitales durante la Navidad, que han sido tradición en España desde hace muchos años.

Uno de los casos paradigmáticos es la forma con la que la Fundación del FC Barcelona ha conseguido impulsar centros XICS a determinados países: México y Brasil. En cada uno de estos, la Fundació ha buscado un partner local, siendo esta una fundación impulsada por uno de sus jugadores del primer equipo en el momento que se crea el centro, sea Rafael Márquez en México o José Edmilson en Brasil. "Los partners locales no implican una relación económica con la Fundació, sino que conocen el territorio y son necesarios para el desarrollo de programas allí. La clave de los XICS es tener un socio local", explica Marc Parramon, responsable de Comunicación de la organización azulgrana. ${ }^{6}$
La campaña que lanzó la Fundació FC Barcelona, junto con Acnur y Nike, bajo el lema Més, también contó con los jugadores del primer equipo de la entidad como principales estandartes publicitarios, e incluso Nike confeccionó una camiseta exclusiva para el equipo, que este lució en la edición del trofeo Joan Gamper del 2009. Posteriormente, esta camiseta se comercializó mediante una edición limitada, junto con toda una línea de productos Més diseñados por Nike con la intención de recabar fondos para programas educativos $y$ deportivos destinados a los niños refugiados (Forns 2008).

En el caso del Espanyol, cuando se realizó el estudio de campo ya se habían organizado once foros. La organización de estos Fòrums Fundació (foros de la fundación), también han contado permanentemente con la presencia de celebridades, no solo del primer equipo de la entidad, sino también de otras vinculadas históricamente con la fundación -por ejemplo, los exentrenadores Javier Clemente y José Antonio Camacho o el exjugador John Lauridsen- o con el mundo del fútbol en general, como el entrenador Johan Cruyff, el periodista deportivo del diario AS Tomás Guasch o la patrona de la fundación del club y nadadora olímpica Gemma Mengual. ${ }^{7}$

6 Información obtenida en la entrevista personal con el responsable de Comunicación de la Fundació FC Barcelona, Marc Parramon, realizada el 26 de octubre del 2009.

7 RCD Espanyol Fundació [en línea]. <http://www.rcdespanyol.cat/>. [Consulta: 27 de diciembre del 2009]. 
Actualmente, la inactividad de la Fundació Privada Catalana de Futbol hace que no podamos tener una lista de celebridades que colaboren con sus proyectos. No obstante, sí que es interesante hacer una referencia final a la importancia que el nuevo entrenador de la selección catalana de fútbol, el holandés Johan Cruyff, ha tenido en la proyección de la federación: la presentación del nuevo entrenador en el Palacio de Congresos de Catalunya, el 9 de noviembre de 2009, contó con la presencia de 150 periodistas acreditados. De todos modos, lo importante del acto es la vinculación que Cruyff ha conseguido con la federación: el dinero que reciba de la federación para desarrollar su cargo como seleccionador estará destinado totalmente a los proyectos sociales de la fundación que lleva su nombre (Agencias, en línea). Ahora, mientras la fundación de la FCF está inactiva, en la web de la federación ya figura un enlace con la Fundación Johan Cruyff.

\section{Conclusiones}

Creemos que las relaciones públicas de plano técnico están muy bien cubiertas desde la actividad comunicativa del club o federación. Sin embargo, los principios estratégicos de relaciones públicas se definen con mayor precisión en el ámbito de actuación de las fundaciones analizadas. Por lo que se refiere al FC Barcelona y al RCD Espanyol, la vocación social de los clu- bes, como forma de diálogo con el entorno y de expresividad de su capacidad de trabajar para el interés común, se percibe ampliamente a través del desarrollo de las actividades de sus fundaciones. Por lo tanto, la estrategia de relaciones públicas está muy bien definida en ambos casos. El proyecto de relaciones públicas de ambos clubes necesita de la fundación para justificar su vocación de institución. Por otro lado, la FCF no necesita de ello, porque nace como órgano colegiado.

Existe un claro proceso de mediatización de los media, puesto que los clubes gozan de sus propios medios de comunicación para influir con más efectividad en la opinión pública y dar su propia visión de los hechos. En este sentido, las fundaciones analizadas son fuentes de contenidos potencialmente publicables que reafirman los valores corporativos de las instituciones que las sustentan. Este proceso no sería posible sin la influencia de las tecnologías de la información y la comunicación, que han facilitado la creación de medios propios como las web, los canales digitales de televisión o los servicios móviles.

Si bien estas tecnologías han permitido crear nuevos canales directos de relación entre organizaciones y públicos, estas también han contribuido a una mayor transparencia de los contenidos, puesto que cuanto más amplia es la red más se amplían las fuentes de información de estos públicos. Los compromisos desarrollados a través 
de las fundaciones tienen que ser, más que nunca, reales y sin presentar dudas sobre sus objetivos. Estamos, sin lugar a dudas, trabajando sobre la actitud auténtica, incluso más que sobre la comunicación que se deriva de ella. Las relaciones públicas desarrollan un rol demostrativo de las posiciones corporativas.

El valor añadido de la organización es, también, el valor añadido del producto, cosa que hace un círculo de comunicación dependiente entre área comercial y área social por la vía de lo corporativo. El área periodística queda como proyector de este circuito. Así como ocurre con las empresas de servicios, FC Barcelona y RCD Espanyol tampoco están al margen de esta premisa, pues tienen, como toda empresa, intereses comerciales (derechos de televisión, merchandising, compraventa de jugadores...) que sustentan la economía de las organizaciones. De esta manera, aunque la razón social de las fundaciones no deja de ser noble, no hay que olvidar que retroalimenta la estrategia comercial de la entidad. Sirva de ejemplo el hecho de que la fundación del FC Barcelona ha sido la principal promotora del eslogan "Más que un club en el mundo" (Badia 2011) o que RCD Espanyol ha usado el Círculo de Amigos del Espanyol para impulsar la primera tienda oficial fuera de Barcelona, en Lloret de Mar.

Las fundaciones contribuyen a la construcción de la personalidad de las organizaciones a las que están asociadas. Son un gran marco de relación que construye actitud colectiva en simbiosis con el entorno, sea a nivel local (por ejemplo, con las escuelas de fútbol del Espanyol o el proyecto de apadrinamiento de jugadores de la Federación) o a nivel internacional (con los proyectos XICS del FC Barcelona). Cuanto mejor está construido el proyecto de relaciones públicas de la entidad, mejor está construido el proyecto de la fundación. Hará falta estudiar, ahora, cómo evolucionan los proyectos de las fundaciones analizadas, sobre todo las dependientes de los clubes FC Barcelona y RCD Espanyol, con las nuevas juntas directivas de Sandro Rosell (2010) o Ramon Condal (2011), respetivamente.

\section{Bibliografía}

Agencias (10 de noviembre del 2009). “Cruyff: ‘No voy a violar el catalán'". La Vanguardia. <http:// www.lavanguardia.es/deportes/ noticias/20091109/53820532743/ cruyff-no-voy-a-violar-el-catalanfederacio-catalana-fc-barcelona-espanyol-daniel-sanchez-llibre-ern. html>. [Consulta: 27 de diciembre del 2009].

Arévalo, M. (2006). Las fundaciones deportivas españolas. Tesis doctoral. Alcalá de Henares: Universidad de Alcalá, Departamento de Didáctica.

BAdiA, J. (2011). “La globalització de les aficions dels clubs de futbol. El caso del FC Barcelona: 'del club 
de Catalunya' al 'més que un club al món'". Tesis para optar el grado de magíster. Bellaterra: Universitat Autònoma de Barcelona, Departament de Periodisme i Ciències de la Comunicació.

Bastenier, M. A. (2001). El blanco móvil. Madrid: Ediciones El País.

Botan, C. H. y V. Hazleton (1989). Public relations theory. Hillsdale, Nueva Jersey: Lawrance Erlbaum Ass.

D'Aprix, R. (1996). Communicating for change. San Francisco: Jossey-Bass Publishers.

Drucker, P. F. (2006). The effective executive. Nueva York: HarperCollins.

- (1980). Managing in turbulent times. Nueva York: Harper \& Row.

FC Barcelona (2009). Fundació. $<$ http://www.fcbarcelona.cat/web/ Fundacio/catala/>. [Consulta: 27 de diciembre del 2009].

-. MES, un nuevo reto solidario. FC Barcelona. <http: //www.fcbarcelona.cat/web/castellano/noticies/club/ temporada08-09/12/n081204106395. html>. [Consulta: 27 de diciembre del 2009].

Forns, V. (2008). "Més que moda". Barça 36. Barcelona: FC Barcelona, 15-16.

Freeman, R. E. (1984). Strategic management: A stakeholders approach. Boston: Pitman.

Freeman, R. E. y J. McVea (2001). “A stakeholder approach to strategic management", en Hitt, M. A.,
Freeman, R. E. y S. H. Jeffrey. Handbook of strategic management. Oxford: Blackwell Publishing, 189-208.

FundACIÓ FC BARCELONA (2009). Memoria 2008-2009. Barcelona: FC Barcelona.

Ginesta, Xavier (2011). “El fútbol y el negocio del entretenimiento global. Los clubes como multinacionales del ocio". Comunicación y Sociedad. Vol. XXIV, núm. 1, 141166. Pamplona: Universidad de Navarra.

-. (2009). Les tecnologies de la informació i la comunicació i l'esport: Una análisis de la primera divisió espanyola de futbol. Tesis para optar el grado de doctor. Bellaterra: Universitat Autònoma de Barcelona, Departament de Mitjans, Comunicació i Cultura.

Ginesta, Xavier y Enric Odeix (2009). Entrevista personal con B. Bafaluy, jefe de Prensa de la Federació Catalana de Futbol, realizada el 22 de octubre del 2009. Barcelona.

- (2009). Entrevista personal con J. Munte, secretario de la Fundació Privada RCD Espanyol, realizada el 5 de noviembre del 2009. Cornellà de Llobregat (España).

- (2009). Entrevista personal con Parramón, M., responsable de comunicación de la Fundació FC Barcelona, realizada el 26 de octubre del 2009. Barcelona.

Grunig, J. et al. (eds.) (1992). Excellence in public relations and communica- 
tion management. Hillsdale, Nueva Jersey: Lawrance Erlbaum Ass.

Helland, K. (2007). Changing sport, changing media. Mass appeal, the sports/media complex and TV sports rights. Nordicom Review, Jubilee Issue. Göteborg: Nordicom, 105-119.

INKPEN, A. (2001). Strategic alliances, en Hitt, M. A., Freeman, R. E. y S. H. Jeffrey (eds.). Handbook of strategic management. Oxford: Blackwell Publishing. pp. 409-432.

Kotter, J. P. (1998). “Leading change: 'Why transformations efforts fail'”, en Collins, J. C. y J. I. Porras (eds.). Harvard Business Review on Change. Boston: Harvard Business School Publishing.

Lefever, K.; Lievens, E. y P. Valcke (20-25 de julio del 2008). Regulating access to sports events on new content platforms. Ponencia presentada en IAMCR World Congress Media and Global Devides. Estocolmo: Universidad de Estocolmo.

Lessinger, E. M. (20-25 de julio del 2008). We don't pick it like Beckham: The German soccer celebrities. Ponencia presentada en IAMCR World Congress Media and Global Devides. Estocolmo: Universidad de Estocolmo.

LEy 50/2002 de Fundaciones (26 de diciembre del 2002). BOE, 27/12/02; c. e. BOE, 17/04/2003. España.

MintzBerg, H. (1995). La estructuración de las organizaciones. Barcelona: Ariel.
Moragas, M. de, et al. (2003). El impacto de Internet en los medios de comunicación y la industria del deporte. Cerdanyola del Vallès: CEO-UAB.

Moss, D. y B. Desanto (2002). Public relations cases. International perspective. Londres: Routledge.

Olabe, F. (2008). "Corporate communication management in Spanish professional football", en ECREA Barcelona 2008. Programme, abstracts, posters and papers. [CD Rom]. Barcelona: InCom-UAB.

Ordeix, E. y J. Duarte (2009). “From public diplomacy to corporate diplomacy:Increasing corporation's legitimacy and influence. American Behavioral Scientist. Vol. 53, núm. 4, 549-564. Nueva York: SAGE Publications.

PARry, J. (10-12 de noviembre del 2003). Ètica esportiva i educació: nous reptes en l'era mediàtica. Ponencia presentada en el Seminario de la Cátedra Internacional de Olimpismo (CIO$\mathrm{UAB}$ ).

Perdiguero, T. G. (2003). La responsabilidad social de las empresas en un mundo global. Barcelona: Anagrama.

Perdiguero, T. G. et al. (2005). La responsabilidad social de las empresas y los nuevos desafíos de la gestión empresarial. Valencia: Universidad de Valencia.

RCD Espanyol. Fundació. <http:// www.rcdespanyol.cat/>. [Consulta: 27 de diciembre del 2009]. 
RCD Espanyol (2007). Memòria 2006/07. Barcelona: RCD Espanyol.

Rowe, D. (2004). Sport, culture and the media. Maidenhead: Open University Press.

-.(2003). "Sportand thereproduction of the global". International Review of the Sociology of Sport 38 (3), 281294. Londres: SAGE Publications.

SpICER, Ch. (1997). Organizational public relations. A political perspective. Mahwah, Nueva Jersey: Lawrance Erlbaum Ass. 